\title{
Dissipations on von Neumann Algebras
}

\author{
C. W. Thompson \\ Department of Mathematics, University of Manchester, Manchester M13 9PL, England
}

\begin{abstract}
We extend a characterisation by Lindblad of complete normal dissipations on hyperfinite von Neumann algebras to general semifinite von Neumann algebras.
\end{abstract}

\section{Introduction}

The time-development of certain quantum systems can be represented by oneparameter semigroups of completely positive maps on the associated $C^{*}$-algebras (see [4] for a discussion of the physical justification for this). When the semigroup is norm-continuous the infinitesimal generator is a bounded linear map on the $C^{*}$-algebra, and Lindblad [4] gives a characterisation of those linear maps which are infinitesimal generators of such semigroups. These he calls complete dissipations.

If we now take a von Neumann algebra $\mathscr{A}$ and look at complete normal dissipations on $\mathscr{A}$, we would like to prove a result corresponding to the theorem that every derivation on a von Neumann algebra is inner. In [4], Lindblad shows that if $\theta: \mathscr{A} \rightarrow \mathscr{A}$ is completely positive then $\gamma_{\theta}: \mathscr{A} \rightarrow \mathscr{A}$ defined by

$$
\gamma_{\theta}(a)=\theta(a)-\frac{1}{2}\{\theta(1) a+a \theta(1)\}
$$

is a complete dissipation on $\mathscr{A}$, and it is clear that $\gamma_{0}$ is normal if and only if $\theta$ is. Definition. A complete dissipation $\gamma$ on a $C^{*}$-algebra $\mathscr{A}$ is called inner if $\gamma-\gamma_{\theta}$ is an inner derivation for some completely positive map $\theta$ on $\mathscr{A}$.

Lindblad shows in [4] that every complete normal dissipation on a hyperfinite von Neumann algebra $\mathscr{A}$ is inner. In [5] he uses the general theory of cohomology of operator algebras to show that the same is true for any type I von Neumann algebra, except that in this case he can only show that the range of the completely positive map $\theta$ is contained in $\mathscr{B}(H)$, where $\mathscr{A}$ is considered as a weak-operator closed subalgebra of $\mathscr{B}(H)$ containing the identity map. However, since any type I von Neumann algebra is injective, there is an expectation from $\mathscr{B}(H)$ onto $\mathscr{A}$, so by the remark at the end of [5] we can choose $\theta$ with range contained in $\mathscr{A}$. 
We show here that every complete normal dissipation on a semifinite von Neumann algebra is inner. The starting point is Proposition 1 of [5], which we state in the next section for completeness.

\section{Preliminary Definitions and Results}

Let $\mathscr{A}$ be a $C^{*}$-algebra with identity.

Definition. A dissipation on $\mathscr{A}$ is a linear map

$\gamma: \mathscr{A} \rightarrow \mathscr{A}$

satisfying, for a in $\mathscr{A}$,

1) $\gamma\left(a^{*}\right)=\gamma(a)^{*}$

2) $\gamma(1)=0$,

3) $\gamma\left(a^{*} a\right) \geqq a^{*} \gamma(a)+\gamma\left(a^{*}\right) a$.

It is called a complete dissipation if

$$
\hat{\gamma}_{n}=\gamma \otimes \mathrm{id}: \mathscr{A} \otimes M_{n} \rightarrow \mathscr{A} \otimes M_{n}
$$

is a dissipation on $\mathscr{A} \otimes M_{n}$ for every $n=1,2, \ldots$, where $M_{n}$ is the $C^{*}$-algebra of $n \times n$ matrices over $\mathbb{C}$ (so $\mathscr{A} \otimes M_{n}$ can be considered as the $C^{*}$-algebra of $n \times n$ matrices over $\mathscr{A}$ ).

Kishimoto shows in [3] that every dissipation on a $C^{*}$-algebra is bounded.

For a dissipation $\gamma$ on a $C^{*}$-algebra $\mathscr{A}$ we define, following Lindblad [5], two related functions, the first from $\mathscr{A} \times \mathscr{A}$ to $\mathscr{A}$ and the second from $\mathscr{A} \times \mathscr{A} \times \mathscr{A}$ to $\mathscr{A}$. They are defined as follows: for $a, b, c$ in $\mathscr{A}$

$$
d(a, b)=d_{\gamma}(a, b)=\gamma(a b)-\gamma(a) b-a \gamma(b)
$$

and

$$
D(a, b, c)=D_{\gamma}(a, b, c)=d(a b, c)-a d(b, c) .
$$

Note that if $\mathscr{A}$ is a von Neumann algebra and $\gamma$ is ultraweakly continuous, then $d$ and $D$ are separately ultraweakly continuous in each variable. Also

$$
d(a, b)=D(a, 1, b) \quad(a, b \in \mathscr{A}) .
$$

The following proposition is Proposition 1 of [5], except for the normality of $\pi$ and $V$, which is easily verified. We can also deduce the normality of $V$ from [7].

Proposition 1. If $\gamma$ is a complete dissipation on a $C^{*}$-algebra $\mathscr{A}$ and $D$ is defined as above, and if $\mathscr{A}$ is considered as a norm-closed algebra of operators on a Hilbert space $H$, containing the identity on $H$, then there is $a^{*}$-representation

$$
\pi: \mathscr{A} \rightarrow \mathscr{B}(K)
$$

of $\mathscr{A}$ on a Hilbert space $K$ and a bounded linear map

$$
V: \mathscr{A} \rightarrow \mathscr{B}(H, K)
$$

such that, for $a, b$, c in $\mathscr{A}$,

$$
D(a, b, c)=V\left(a^{*}\right)^{*} \pi(b) V(c)
$$


and

$$
V(a b)=V(a) b+\pi(a) V(b) .
$$

If $\mathscr{A}$ is a von Neumann algebra and is ultraweakly closed in $\mathscr{B}(H)$ and $\gamma$ is normal then $\pi$ and $V$ can be chosen to be normal (i.e. continuous in the ultraweak topologies on $\mathscr{B}(H)$ and $\mathscr{B}(H, K))$.

For the remainder of the paper we assume, unless otherwise stated, that $\mathscr{A}$ is a von Neumann algebra, considered as a weak-operator closed subalgebra of operators on a Hilbert space $H$, containing the identity on $H$, and that $\gamma$ is a complete normal dissipation on $\mathscr{A}$.

Define

$$
\Lambda_{0}=\{\pi(a) V(b) c: a, b, c \in \mathscr{A}\} \subseteq \mathscr{B}(H, K),
$$

where $\pi, V, K$ are as in Proposition 1. If

$$
x=\pi\left(a_{1}\right) V\left(b_{1}\right) c_{1}, y=\pi\left(a_{2}\right) V\left(b_{2}\right) c_{2}
$$

are general elements in $\Lambda_{0}$, then

$$
\begin{aligned}
y^{*} x & =c_{2}^{*} V\left(b_{2}\right)^{*} \pi\left(a_{2}^{*}\right) \pi\left(a_{1}\right) V\left(b_{1}\right) c_{1} \\
& =c_{2}^{*} V\left(b_{2}\right)^{*} \pi\left(a_{2}^{*} a_{1}\right) V\left(b_{1}\right) c_{1} \\
& =c_{2}^{*} D\left(b_{2}^{*}, a_{2}^{*} a_{1}, b_{1}\right) c_{1} \in \mathscr{A} .
\end{aligned}
$$

Now let $\Lambda$ be the weak-operator closed linear span of $\Lambda_{0}$.

Lemma 2. (i) $y^{*} x \in \mathscr{A}$ for every $x, y$ in $\Lambda$;

(ii) $\pi(a) x$ and $x a \in \Lambda$ for every $x \in \Lambda, a \in \mathscr{A}$.

Proof. Let $\Lambda_{1}$ be the linear span of $\Lambda_{0}$ and let

$$
x=\sum_{i} \lambda_{i} x_{i}, y=\sum_{j} \mu_{j} y_{j}
$$

be general elements of $\Lambda_{1}\left(\lambda_{i}, \mu_{j} \in \mathbb{C}, x_{i}, y_{j} \in \Lambda_{0}\right)$. Then

$$
\begin{aligned}
y^{* x} & =\left(\sum_{j} \bar{\mu}_{j} y_{j}^{*}\right)\left(\sum_{i} \lambda_{i} x_{i}\right) \\
& =\sum_{i, j} \lambda_{i} \bar{\mu}_{j} y_{j}^{*} x_{i} \in \mathscr{A}
\end{aligned}
$$

by the previous calculation.

Now let $x, y \in \Lambda$ and choose nets $\left(x_{\alpha}\right),\left(y_{\beta}\right)$ in $\Lambda_{1}$, converging in the weakoperator topology to $x, y$ respectively. Since $\mathscr{A}$ is weak-operator closed in $\mathscr{B}(H)$ and multiplication is separately weak-operator continuous, fixing $\beta$ we have

$$
y_{\beta}^{*} x=\lim _{\alpha} y_{\beta}^{*} x_{\alpha} \in \mathscr{A} .
$$

Now using the fact that the *-operation is weak-operator continuous we obtain

$$
y^{*} x=\lim _{\beta} y_{\beta}^{*} x \in \mathscr{A} \text {. }
$$

The proof of (ii) is similar and is omitted. 
Note. Since $\Lambda$ is a weak-operator closed subspace of $\mathscr{B}(H, K)$ it has a predual $\Lambda_{*}$, and with respect to this predual it becomes a dual normal $\mathscr{A}$-module in the sense of $[8$, p. 404]. Further, by (i) of Lemma 2 we can define an $\mathscr{A}$-valued "inner product" on $\Lambda$ by

$$
(x, y) \mapsto y^{*} x \quad(x, y \in \Lambda)
$$

and $A$ thus becomes a right Hilbert $\mathscr{A}$-module [6]. It can be shown that the dual normal module structure on $A$ implies that it is a self-dual right Hilbert $\mathscr{A}$-module in the sense of Paschke [6]. By Proposition $1, V$ ia a derivation of $\mathscr{A}$ into $A$. In what follows we implicitly use the $\mathscr{A}$-module structure on $A$, and in particular the $\mathscr{A}$-valued inner product.

\section{The Main Results}

The proof of the following proposition is an adaptation of the proof by Johnson and Ringrose ([2] or [9, Theorem 4.1.6]) that every derivation on a von Neumann algebra is inner.

The proof easily generalises to prove that every derivation on a dual normal Hilbert module over a semi-finite von Neumann algebra is inner.

Proposition 3. Let $\mathscr{A}$ be a semi-finite von Neumann algebra. Then with the same notation and assumptions as in the previous section, there is a $\hat{V} \in \Lambda$ with $\|\hat{V}\| \leqq\|V\|$ such that for a in $\mathscr{A}$,

$$
V(a)=\hat{V} a-\pi(a) \hat{V} .
$$

Proof. We write $\mathscr{A}^{u}$ for the group of unitary elements of $\mathscr{A}$. For $u$ in $\mathscr{A}^{u}$ define as map

$$
T_{u}: \Lambda \rightarrow \Lambda
$$

by

$$
T_{u}(x)=\pi(u) x u^{*}+V(u) u^{*} \quad(x \in \Lambda) .
$$

For $u, v$ in $\mathscr{A}^{u}$ and $x$ in $\Lambda$,

$$
\begin{aligned}
T_{u}\left(T_{v}(x)\right) & =T_{u}\left[\pi(v) x v^{*}+V(v) v^{*}\right] \\
& =\pi(u)\left[\pi(v) x v^{*}+V(v) v^{*}\right] u^{*}+V(u) u^{*} \\
& =\pi(u v) x(u v)^{*}+[\pi(u) V(v)+V(u) v](u v)^{*} \\
& =\pi(u v) x(u v)^{*}+V(u v)(u v)^{*} \\
& =T_{u v}(x),
\end{aligned}
$$

so $T_{u} T_{v}=T_{u v}$ for $u, v$ in $\mathscr{A}^{u}$.

Let $\Delta$ be the collection of non-empty, weak-operator closed convex sets $K$ of $A$ satisfying

1) $T_{u}(K) \cong K \quad\left(u \in \mathscr{A}^{u}\right)$,

and

2) $\sup \{\|x\|: x \in K\} \leqq\|V\|$. 
For $u$ in $\mathscr{A}^{u},\left\|T_{u}(0)\right\|=\left\|V(u) u^{*}\right\| \leqq\|V\|, T_{u}(0) \in \Lambda$, and

$$
T_{u}\left(\left\{T_{v}(0): v \in \mathscr{A}^{u}\right\}\right)=\left\{T_{u v}(0): v \in \mathscr{A}^{u}\right\}=\left\{T_{v}(0): v \in \mathscr{A}^{u}\right\},
$$

so the weak-operator closure of the convex hull of $\left\{T_{v}(0): v \in \mathscr{A}^{u}\right\}$ is a member of $\Delta$, and $\Delta$ is non-empty.

Order $\Delta$ by inclusion. Using the fact that weak-operator closed bounded sets in $A$ are weak-operator compact [since $A$ is weak-operator closed in $\mathscr{B}(H, K)$ ], we can easily see that each chain in $\Delta$ has a lower bound, namely the intersection of all members of the chain. So by Zorn's lemma, $\Delta$ has a minimal element $K_{0}$.

If $x, y \in K_{0}$ and $u \in \mathscr{A}^{u}$

$$
\pi(u)(x-y) u^{*}=T_{u}(x)-T_{u}(y) \in K_{0}-K_{0},
$$

so $K_{0}-K_{0}$ is invariant under the mappings

$$
\Phi^{u}: z \mapsto \pi(u) z u^{*}(z \in \Lambda)
$$

for each $u \in \mathscr{L}^{u}$.

Firstly assume that $\mathscr{A}$ is a finite, countably-decomposable von Neumann algebra and therefore has a faithful tracial state $\tau$. Define an inner product $\langle., .\rangle_{\tau}$ on $\Lambda$ by

$$
\langle x, y\rangle_{\tau}=\tau\left(y^{*} x\right) \quad(x, y \in \Lambda) .
$$

This is well-defined by Lemma 2(i). We write

$$
\|x\|_{\tau}=\langle x, x\rangle_{\tau}^{1 / 2} \quad(x \in \Lambda) \text {. }
$$

We want to show that $K_{0}-K_{0}=\{0\}$, so conversely assume that there is a non-zero $c=a-b$ with $a, b$ in $K_{0}$. Let

$$
\lambda=\sup \left\{\|x\|_{\tau}: x \in K_{0}\right\} \text {. }
$$

If $x \in K_{0}$, the weak-operator closure of the convex hull of $\left\{T_{u}(x): u \in \mathscr{A}^{u}\right\}$ is a member of $\Delta$ and contained in $K_{0}$ (by the invariance of $K_{0}$ relative to the $T_{u}$ ), so by minimality it must be equal to $K_{0}$. So taking $x=\frac{1}{2}(a+b)$ and $s>0$ we can find a $u \in \mathscr{A}^{u}$ such that

$$
\left\|T_{u}\left(\frac{a+b}{2}\right)\right\|_{\tau}>\lambda-\varepsilon .
$$

Since $\left\|T_{u}(a)\right\|_{\tau} \leqq \lambda,\left\|T_{u}(b)\right\|_{\tau} \leqq \lambda$, by the parallelogram law,

$$
\begin{aligned}
\left\|\frac{1}{2}\left(T_{u}(a)-T_{u}(b)\right)\right\|_{\tau}^{2}= & \frac{1}{2}\left(\left\|T_{u}(a)\right\|_{\tau}^{2}+\left\|T_{u}(b)\right\|_{\tau}^{2}\right) \\
& -\left\|\frac{1}{2}\left(T_{u}(a)+T_{u}(b)\right)\right\|_{\tau}^{2} \\
& \leqq \frac{1}{2}\left(\lambda^{2}+\lambda^{2}\right)-(\lambda-\varepsilon)^{2} \\
& =2 \lambda \varepsilon-\varepsilon^{2}
\end{aligned}
$$

since $\frac{1}{2}\left(T_{u}(a)+T_{u}(b)\right)=T_{u}\left(\frac{a+b}{2}\right)$. 
But on the other hand

$$
\begin{aligned}
\left\|T_{u}(a)-T_{u}(b)\right\|_{\tau}^{2} & =\left\|\pi(u)(a-b) u^{*}\right\|_{\tau}^{2} \\
& =\tau\left(u(a-b)^{*}(a-b) u^{*}\right) \\
& =\tau\left((a-b)^{*}(a-b)\right) \quad \text { (since } \tau \text { is tracial) } \\
& =\|a-b\|_{\tau}^{2},
\end{aligned}
$$

so letting $\varepsilon \rightarrow 0$ we get $\|a-b\|_{\tau}^{2}=0$ and $a-b=c=0$ (since $\tau$ is faithful), a contradiction. Hence $K_{0}-K_{0}=\{0\}$, and $K_{0}$ consists of a single point $\hat{V}$ say. Since $K_{0}$ is invariant under each $T_{u}$,

$$
[\pi(u) \hat{V}+V(u)] u^{*}=\hat{V} \quad\left(u \in \mathscr{A}^{u}\right)
$$

and rearranging

$$
V(u)=\hat{V} u-\pi(u) \hat{V} \quad\left(u \in \mathscr{A}^{u}\right) .
$$

But $\mathscr{A}^{u}$ linearly generates $\mathscr{A}$, so

$$
V(a)=\hat{V} a-\pi(a) \hat{V} \quad(a \in \mathscr{A}) .
$$

Note that by construction $\|\hat{V}\| \leqq\|V\|$ and $\hat{V} \in \Lambda$.

Now let $\mathscr{A}$ be any semifinite von Neumann algebra. For a countablydecomposable finite projection $e$ in $\mathscr{A}$ define

$$
V_{e}: e \mathscr{A} e \rightarrow \mathscr{B}(e H, \pi(e) K)
$$

by

$$
V_{e}(e a e)=\pi(e) V(e a e) e .
$$

e⿻A e is a finite countably-decomposable von Neumann algebra, so by the first half of the proof there is a $\hat{V}_{e} \in \pi(e) \Lambda e$ with $\left\|\hat{V}_{e}\right\| \leqq\left\|V_{e}\right\| \leqq\|V\|$ and

$$
V_{e}(e a e)=\hat{V}_{e} e a e-\pi(e a e) \hat{V}_{e} \quad(a \in \mathscr{A}) \text {. }
$$

Now let $\left(e_{x}\right)_{x \in I}$ be an increasing directed set of finite countably decomposable projections with supremum 1 (see corresponding proof in [2] for a proof of the existence of such a net). Then for each $\alpha \in I$ there is a $\hat{V}_{\alpha}=\hat{V}_{e_{2}}$ with $\left\|\hat{V}_{\alpha}\right\| \leqq\|V\|$ and

$$
V_{e_{x}}\left(e_{x} a e_{\alpha}\right)=\hat{V}_{\gamma} e_{\alpha} a e_{\alpha}-\pi\left(e_{\gamma} a e_{\alpha}\right) \hat{V}_{x} \quad(a \in \mathscr{A}) .
$$

Also $\hat{V}_{x} \in \pi\left(e_{x}\right) \Lambda e_{x} \subseteq \Lambda$. By the weak-operator compactness of bounded sets in $\Lambda$ we can find a cofinal convergent subset of $\left(\hat{V}_{x}\right)_{x \in I}$, and so we may assume that $\left(V_{x}\right)_{x \in I}$ is weak-operator convergent to an element $V$ in $A$ with $\|\hat{V}\| \leqq\|V\|$ (the subnet of projections has supremum 1 since it is cofinal).

$$
\begin{aligned}
& \text { If } \beta \leqq \alpha, e_{\beta} \leqq e_{\alpha} \text { so } \\
& V_{e_{\alpha}}\left(e_{\beta} a e_{\beta}\right)=\hat{V}_{\alpha} e_{\beta} a e_{\beta}-\pi\left(e_{\beta} a e_{\beta}\right) \hat{V}_{\alpha} \quad(a \in \mathscr{A}) .
\end{aligned}
$$

Letting $\alpha \rightarrow \infty$ and noting that $V_{e_{\alpha}}\left(e_{\beta} a e_{\beta}\right)=\pi\left(e_{\alpha}\right) V\left(e_{\beta} a e_{\beta}\right) e_{\alpha} \rightarrow V\left(e_{\beta} a e_{\beta}\right)$ in the weakoperator topology, we get

$$
V\left(e_{\beta} a e_{\beta}\right)=\hat{V} e_{\beta} a e_{\beta}-\pi\left(e_{\beta} a e_{\beta}\right) \hat{V} \quad(a \in \mathscr{A}) .
$$


Now let $\beta \rightarrow \infty$, so $e_{\beta} a e_{\beta}$ converges to $a$ ultraweakly and in the weak-operator topology (the two topologies coincide on bounded sets); then using the normality of $\pi$ and $V$,

$$
\begin{aligned}
V(a) & =\lim _{\beta} V\left(e_{\beta} a e_{\beta}\right) \\
& =\lim _{\beta}\left[\hat{V} e_{\beta} a e_{\beta}-\pi\left(e_{\beta} a e_{\beta}\right) \hat{V}\right] \\
& =\hat{V} a-\pi(a) \hat{V} \quad(a \in \mathscr{A})
\end{aligned}
$$

as required.

We can now easily deduce the main result of the paper.

Theorem 4. Every complete normal dissipation on a semi-finite von Neumann algebra is inner.

Proof. With the same notation as before, put

$$
\theta(a)=\hat{V}^{*} \pi(a) \hat{V} \quad(a \in \mathscr{A}) .
$$

By Lemma $2 \theta(\mathscr{A}) \subseteq \mathscr{A}$ and by [10] $\theta$ is completely positive. A straightforward calculation gives

$$
\begin{aligned}
d_{\gamma_{\theta}}(a, b) & =\left(\hat{V} a^{*}-\pi\left(a^{*}\right) \hat{V}\right)^{*}(\hat{V} b-\pi(b) \hat{V}) \\
& =V\left(a^{*}\right)^{*} V(b) \\
& =D_{\gamma}(a, 1, b) \\
& =d_{\gamma}(a, b) \quad(a, b \in \mathscr{A}),
\end{aligned}
$$

so

$$
d_{(\gamma-\gamma \theta)}(a, b)=d_{\gamma}(a, b)-d_{\gamma_{\theta}}(a, b)=0 \quad(a, b \in \mathscr{A}),
$$

that is, $\gamma-\gamma_{\theta}$ is a derivation on $\mathscr{A}$. But every derivation on a von Neumann algebra is inner, so $\gamma$ is inner.

Note. If $\mathscr{A}$ is a non-hyperfinite type III von Neumann algebra we do not know whether every complete normal dissipation on $\mathscr{A}$ is inner. However Christensen has proved in [1] that if $\mathscr{A}$ is considered as a weakly-closed subalgebra of $\mathscr{B}(H)$, containing the identity on $H$, and $V: \mathscr{A} \rightarrow \mathscr{B}(H)$ is a derivation, then there is a $\hat{V} \in \mathscr{B}(H)$ such that

$$
V(a)=\hat{V} a-a \hat{V} \quad(a \in \mathscr{A}) .
$$

Using this result we can easily deduce that if $\pi: \mathscr{A} \rightarrow \mathscr{B}(K)$ is a normal *-representation of $\mathscr{A}$ on a Hilbert space $K$ and $V: \mathscr{A} \rightarrow \mathscr{B}(H, K)$ is a derivation (where $\mathscr{B}(H, K)$ is an $\mathscr{A}$-module in the obvious way), then there is a $\hat{V} \in \mathscr{B}(H, K)$ such that

$$
V(a)=\hat{V} a-\pi(a) \hat{V}
$$


[consider $\mathscr{B}(H, K)$ as a submodule of $\mathscr{B}(H \oplus K)$ in the obvious way]. Combining this result with Proposition 1 we obtain the following:

If $\gamma$ is a complete normal dissipation on a type III von Neumann algebra $\mathscr{A} \cong \mathscr{B}(H)$ then there is a completely positive map $\theta: \mathscr{A} \rightarrow \mathscr{B}(H)$ such that $\gamma_{\theta}$ is a complete normal dissipation on $\mathscr{A}$ and $\gamma-\gamma_{\theta}$ is a derivation on $\mathscr{A}$ (which is therefore inner).

Acknowledgements. I would like to thank Dr. E. C. Lance for suggesting this problem to me and giving me guidance in my effort towards its solution. I would also like to thank Professor J. R. Ringrose for bringing to my attention the paper by Christensen [1].

\section{References}

1. Christensen, E.: Extensions of derivations (to appear)

2. Johnson, B.E., Ringrose,J. R.: Derivations of operator algebras and discrete group algebras. Bull. London Math. Soc. 1, $70-74$ (1969)

3. Kishimoto, A.: Dissipations and derivations. Commun. math. Phys. 47, 25-32 (1976)

4. Lindblad,G.: On the generators of quantum dynamical semigroups. Commun. math. Phys. 48, $119-130(1976)$

5. Lindblad,G.: Dissipative operators and cohomology of operator algebras. Letters Math. Phys. 1, $219-224(1976)$

6. Paschke,W.L.: Inner product modules over $B^{*}$-algebras. Trans. Am. Math. Soc. 182, $443-468$ (1973)

7. Ringrose,J. R. : Automatic continuity of derivations of operator algebras. J. London Math. Soc. 5, $432-438(1972)$

8. Ringrose,J. R. : Cohomology of operators algebras. Lectures on operator algebras. Lecture notes in mathematics, Vol. 247. Berlin-Heidelberg-New York: Springet 1972

9. Sakai,S. $C^{*}$-algebras and $W^{*}$-algebras. Berlm-Heidelberg-New York: Springer 1971

10. Stinespring, S.: Positive functions on $C^{*}$-algebras. Proc. Am. Math. Soc. 6, 211-216 (1955)

Communicated by H. Araki

Received December 12, 1977 